\section{Carbon nanoparticles and oxidative stress: could an injection stop brain damage in minutes?}

\author{
"PEG-functionalized hydrophilic carbon clusters have been shown to \\ carry out therapeutic functions in preclinical models that have been \\ unattainable from enzymes or small molecule treatment..."
}

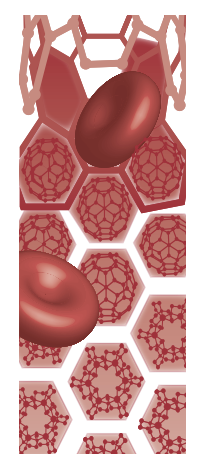

\author{
Lizanne G Nilewski \\ Department of Chemistry \\ \& Department of Materials Science \\ \& NanoEngineering, Rice University, \\ 6100 Main Street, Houston, \\ TX 77005, USA \\ William KA Sikkema \\ Department of Chemistry \\ \& Department of Materials Science \\ \& NanoEngineering, Rice University, \\ 6100 Main Street, Houston, \\ TX 77005, USA
}

\section{Challenges in treatment of oxidative stress}

Reactive oxygen species (ROS), which include superoxide, nitric oxide, hydroxyl radicals, nitrogen dioxide and peroxynitrite, play a crucial role in vital processes such as blood pressure regulation, cell migration, neurotransmission, immune regulation, microorganism defense and smooth muscle relaxation [1]. Under normal conditions in healthy systems, ROS are regulated by enzymes including superoxide dismutase (SOD) and catalase, as well as by small molecule natural antioxidants such as glutathione, ascorbic acid, coenzyme Q10, vitamin A and vitamin E. The overproduction of ROS leads to a condition known as oxidative stress, and at elevated concentrations, ROS readily cause oxidative damage to biomolecules including DNA, lipids, proteins and enzymes. Extreme levels of oxidative stress lead to extensive cellular damage, and these elevated levels of ROS often accompany acute injuries such as traumatic brain injury, inflammatory response and stroke as well as chronic diseases including cancer, cardiovascular dysfunction, rheumatoid arthritis, Parkinson's disease and Alzheimer's disease [1]. While natural antioxidants can regulate and alleviate oxidative stress to some degree, they have little effect on extremely elevated ROS levels, and this is due to their specific mechanisms of action. Many naturally occurring antioxidants and antioxidant enzymes act through chain reactions of radical transfers, often requiring cofactors, or need to act in concert with other antioxidants to be effective. For example, SOD converts two molecules of superoxide into oxygen and hydrogen peroxide, but it requires the presence of another enzyme, catalase, to complete the catalytic cycle. Additionally, as with SOD, many natural antioxidants can only quench one or two reactive species per molecule before requiring regeneration. Thus, these natural antioxidants can be easily overwhelmed by the highly elevated levels of ROS during disease or traumatic injury, and the effects are exacerbated when the trauma is accompanied by hemorrhagic shock. Overall, these limitations of natural antioxidants explain why most studies of antioxidant therapies in a clinical setting show little to no benefit in disease or injury treatment [2]. The benefit there is from antioxidant therapy is mostly apparent in models where the antioxidant is administered before the injury takes place, and few have been shown to have any significant affect postinjury. There is therefore a distinct need to develop synthetic antioxidant treatments that renormalize elevated levels of oxidative stress, and especially therapies that can be administered after an injury has taken place. An optimal antioxidant would be able to regenerate itself without needing a cascade of other enzymes or small molecules to complete the cycle, rapidly react with multiple ROS, be able to quench reactive species without creating potentially harmful byproducts, not react with helpful radicals, such as the vasodilator nitric oxide, show efficacy when administered postinjury,

\section{Thomas A Kent}

Department of Neurology, Baylor College of Medicine, One Baylor Plaza, Houston, TX 77030, USA and

Center for Translational Research on Inflammatory Diseases, Michael E DeBakey VA Medical Center, 2002 Holcombe Boulevard, Houston, TX 77030, USA

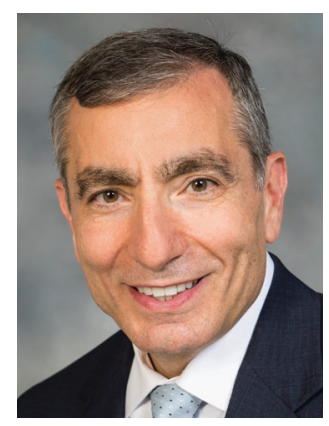

James M Tour Author for correspondence: Department of Chemistry \& Department of Materials Science \& NanoEngineering, Rice University, 6100 Main Street, Houston, TX 77005, USA

\section{Future $\because: \%$ Medicine part of}


and be nontoxic and readily cleared post elevated levels of ROS. If such an antioxidant could be realized, it could act orthogonally to the cell's natural antioxidant system and thereby mitigate the ROS independently, allowing it to be effective when the cell's antioxidant system is compromised.

\section{"Encouragingly, these PEG-functionalized hydrophilic carbon cluster carbon nanoparticles show antioxidant activity that has not been realized for any enzymatic system or small molecule therapy."}

\section{Carbon nanoparticle-based antioxidants}

Our team has discovered nontoxic carbon nanoparticlebased antioxidants that have reactivity profiles toward ROS that fit with the optimal profile. The nanoparticles are called PEG-functionalized hydrophilic carbon clusters (PEG-HCCs). These are $3 \times 40 \mathrm{~nm}$ highly edgeoxidized PEGylated fragments of pi-conjugated monoatomic layer graphene-like domains [3]. The PEG-HCCs are nontoxic, and they have a half-life of 2 to $3 \mathrm{~h}$ in vivo. Encouragingly, these PEG-HCC carbon nanoparticles show antioxidant activity that has not been realized for any enzymatic system or small molecule therapy.

Originally, PEG-HCCs were used as passive drug delivery nanovectors, where the hydrophobic network of $\mathrm{sp}^{2}$ carbons acts as a core that can physisorb similarly hydrophobic drugs and targeting antibodies. The oxidation on the edges of the HCCs and the PEG moieties allows the entire complex to be water or phosphate-buffered saline soluble, permitting intravenous delivery of the drug/PEG-HCC combination where the drugs are physisorbed to the conjugated domains. The use of paclitaxel/PEG-HCCs has been shown to be as effective as the standard drug formulation $\mathrm{Taxol}^{\mathrm{TM}}[4]$, the latter requiring patient premedication of high-dose corticosteroids to avoid clinical acute hypersensitivity to the surfactant Cremophor EL. The Cremophor EL is used to maintain water solubility of the otherwise insoluble paclitaxel. The PEG-HCCs can also be used in an antibody/ drug/PEG-HCC formulation where both the drug and the antibody are physisorbed to the HCC core. Using an EGFR antibody physisorbed to the surface of the PEG-HCCs, we are able to selectively target an EGFR+ tumor over an EGFR- tumor in a single mouse [5]. We have also found that using short peptides that are covalently attached to the PEG termini, drug/peptide-PEG-HCC combinations are effective at treating several cancer types [6]. Thus, PEG-HCCs can be targeted through noncovalent antibody delivery, or through covalently bound peptide recognition schemes, thereby minimizing nanoparticle dosages.
Extending beyond the passive drug delivery applications of the PEG-HCCs, they can act as powerful active antioxidants due to the extended pi-conjugated domains of the HCCs. Like some fullerene derivatives which also contain extended pi-conjugation [7], PEG-HCCs are antioxidants that quench ROS, such as superoxide and hydroxyl radicals, in cell-free systems [3], in vitro and in vivo $[4-5,8]$. However, PEG-HCCs act much more quickly than any fullerene-based system. The turnover rate of superoxide to oxygen by PEG-HCCs was measured to be $>20,000 \mathrm{~s}^{-1}$, which is higher than most single-active-site enzymes, suggesting that the PEG-HCCs have multiple active sites [9]. This allows the PEGHCCs to be effective even when administered after the traumatic event, making them clinically unique [10].

\section{Applications to brain injury}

In cases of extreme oxidative stress in the brain, for example, in conditions of stroke or traumatic brain injury accompanied by hemorrhagic shock, the rapid kinetics of the PEG-HCCs allows them to act quickly to stop brain damage. In addition to the elevated levels of superoxide produced during the initial injury, a second wave of superoxide is produced by cells as the injury is being treated by reperfusion of blood. One contribution to this wave is the buildup of hypoxanthine during hypoxia which suddenly reacts via xanthine oxidase with the oxygen in reperfused blood to produce superoxide [11]. This second spike of superoxide can be addressed clinically by administration of the high-capacity antioxidants PEG-HCCs directly before reperfusion [12]. Indeed, in a rat model of traumatic brain injury accompanied by hemorrhagic shock, measurements taken after PEG-HCC treatment and blood reperfusion showed that animals treated with PEGHCCs had normal levels of superoxide in the brain and vasculature, while those not treated with PEGHCCs had as much as double the levels of superoxide. Additionally, the PEG-HCCs did not react with the essential nitric oxide. Rather, it was found that PEGHCCs normalized nitric oxide levels in the treatment group, while in the controls, the nitric oxide levels were significantly lowered, presumably due to reaction between nitric oxide and the excess superoxide to form the highly deleterious peroxynitrite [10]. Preliminarily, PEG-HCCs have been extended to stroke models where the PEG-HCCs are delivered before clearance of the clot, potentially mitigating superoxide buildup during blood reperfusion to the brain.

\section{Future perspective}

Injury and disease due to oxidative stress extend far past the brain. Cancer becomes more metastatic under oxidative stress conditions [13], inflammation and 
superoxide are intimately coupled [11,14], and superoxide plays a role in numerous chronic disorders such as diabetes, Parkinson's and Alzheimer's diseases [1,15]. PEG-HCCs have been shown to carry out therapeutic functions in preclinical models that have been unattainable from enzymes or small molecule treatment, and this could be a harbinger for the use of active carbon nanoparticle-based therapies. Therefore, PEGHCCs could soon enjoy a prominent place in the treatment of acute injuries and chronic disorders.

Financial \& competing interests disclosure

The authors' current support for PEG-HCC research is from the NIH: 5 R21 DK093802-02 and 5 R21 NS084290-02. Pre-

\section{References}

Papers of special note have been highlighted as:

- of interest; $\bullet \bullet$ of considerable interest

1 Valko M, Leibfritz D, Moncol J, Cronin MTD, Mazur $\mathrm{M}$, Telser J. Free radicals and antioxidants in normal physiological functions and human disease. Int. J. Biochem. Cell Biol. 39, 44-84 (2007).

2 Slemmer JE, Shacka JJ, Sweeney MI, Weber JT. Antioxidants and free radical scavengers for the treatment of stroke, traumatic brain injury and aging. Curr. Med. Chem. 15, 404-414 (2008).

3 Lucente-Schultz RM, Moore VC, Leonard AD et al. Antioxidant single-walled carbon nanotubes. J. Am. Chem. Soc. 131(9), 3934-3941 (2009).

4 Berlin JM, Leonard AD, Pham TT et al. Effective drug delivery, in vitro and in vivo, by carbon-based nanovectors noncovalently loaded with unmodified paclitaxel. ACS Nano 4(8), 4621-4636 (2010).

- Covers the drug delivery and toxicity aspects of the PEG-functionalized hydrophilic carbon clusters (PEG-HCCs).

5 Sano D, Berlin JM, Pham TT et al. Noncovalent assembly of targeted carbon nanovectors enables synergistic drug and radiation cancer therapy in vivo. ACS Nano 6(3), 2497-2505 (2012).

- Covers the targeting of PEG-HCCs as delivery vehicles.

6 Sharpe MA, Marcano DC, Berlin JM, Widmayer MA, Baskin DS, Tour JM. Antibody-targeted nanovectors for the treatment of brain cancers. ACS Nano 6(4), 3114-3120 (2012).

7 Ali SS, Hardt JI, Quick KL et al. A biologically effective fullerene (C60) derivative with superoxide dismutase mimetic properties. Free Radic. Biol. Med. 37(8), 1191-1202 (2004). vious Department of Defense support was from the Mission Connect Mild Traumatic Brain Injury Consortium (W81XWH-08-2-0141 [TA Kent], W81XWH-08-2-0143 [JM Tour]) and the Alliance for NanoHealth (W81XWH-09-02-0139). JM Tour and TA Kent are shareholders in a company that has licensed the PEG-HCC therapeutic technology from Rice University. Conflicts of interests are overseen by the appropriate offices at the respective academic institutions. The authors have no other relevant affiliations or financial involvement with any organization or entity with a financial interest in or financial conflict with the subject matter or materials discussed in the manuscript apart from those disclosed.

No writing assistance was utilized in the production of this manuscript.

8 Marcano DC, Bitner BR, Berlin JM et al. Design of Poly(ethylene glycol)-functionalized hydrophilic carbon clusters for targeted therapy of cerebrovascular dysfunction in mild traumatic brain injury. J. Neurotrauma $30(9)$, 789-796 (2013).

9 Samuel ELG, Marcano DC, Berka V et al. Highly efficient conversion of superoxide to oxygen using hydrophilic carbon clusters. Proc. Natl Acad. Sci. 112(8), 2343-2348 (2015).

-. The kinetics of the PEG-HCC reaction with superoxide are discussed in detail.

10 Bitner BR, Marcano DC, Berlin JM et al. Antioxidant carbon particles improve cerebrovascular dysfunction following traumatic brain injury. ACS Nano 6(9), 8007-8014 (2012).

-• The use of PEG-HCCs in vivo in a traumatic brain injury/hemorrhagic shock model.

11 McCord JM, Ranjan RS. The pathophysiology of superoxide: roles in inflammation and ischemia. Can. J. Physiol. Pharmacol. 60 (11), 1346-1352 (1982).

12 Fabian RH, DeWitt DS, Kent TA. In vivo detection of superoxide anion production by the brain using a cytochrome c electrode. J. Cereb. Blood Flow Metab. 15, 242-247 (1995).

13 LeBleu VS, O'Connell JT, Gonzalez Herrera KN et al. PGC-1 $\alpha$ mediates mitochondrial biogenesis and oxidative phosphorylation in cancer cells to promote metastasis. Nat. Cell Biol. 16(10), 992-1003 (2014).

14 Guzik TJ, Korbut R, Adamek-Guzik T. Nitric oxide and superoxide in inflammation and immune regulation. J. Physiol. Pharmacol. 54, 469-487 (2003).

15 Hildeman DA, Mitchell T, Kappler J, Marrack P. T cell apoptosis and reactive oxygen species. J. Clin. Invest. 111(5), 575-581 (2003). 